\title{
Electron energy loss spectroscopy as a probe of two-dimensional photonic crystals
}

\author{
F. J. García de Abajo, ${ }^{1, *}$ A. Rivacoba, ${ }^{1}$ N. Zabala, ${ }^{1,2}$ and P. M. Echenique ${ }^{1}$ \\ ${ }^{1}$ Centro Mixto CSIC-UPV/EHU and Donostia International Physics Center (DIPC), Aptdo. 1072, 20080 San Sebastián, Spain \\ ${ }^{2}$ Departamento de Electricidad y Electrónica, Facultad de Ciencias, UPV/EHU, Aptdo. 644, 48080 Bilbao, Spain
}

(Received 3 June 2003; revised manuscript received 24 July 2003; published 19 November 2003)

\begin{abstract}
The band structure and density of states of two-dimensional photonic crystals are shown to be directly related to the loss spectra of electrons moving parallel to the direction of translational symmetry. Both broad electron beams and focused beams passing through crystals composed of cylindrical holes in a dielectric host are considered. Energy losses originate in Cherenkov light emission by coupling to radiation modes of the crystal. The angular distribution of transmitted electrons is shown to map quite closely the band structure of the crystal when broad beams are employed, and in particular, Van Hove singularities give rise to peaks in the loss probability. Finally, the degree of convergence towards the infinite crystal results is studied for finite crystals of increasing size.
\end{abstract}

DOI: 10.1103/PhysRevB.68.205105

PACS number(s): 79.20.- m, 42.70.Qs, 41.60.Bq

\section{INTRODUCTION}

The optical properties of bulk materials and small particles have been routinely determined by electron energy loss spectroscopy (EELS). ${ }^{1,2}$ This is usually performed in electron microscopes using fast electrons $(20-300 \mathrm{keV})$ that can be focused on spots below $1 \mathrm{~nm}$ in diameter. ${ }^{3}$ The passing electrons perturb the sample with their electric field, which is evanescent in vacuum, where it extends a distance away from the trajectory of the order of the electron velocity divided by the excitation frequency, $v / \omega$. This makes of EELS an excellent technique to probe the excitation modes of the sample near the electron trajectory up to distances below $\sim v / \omega$.

When the electrons move inside a transparent material or close to its boundary, Cherenkov radiation can be produced if the electron velocity exceeds the speed of light in the medium. ${ }^{4}$ However, the intensity of the emitted light can be strongly modulated by the presence of boundaries. In particular, the Cherenkov radiation pattern suffers severe modifications in a photonic crystal, as reported recently for twodimensional (2D) crystals and electron trajectories perpendicular to the direction of translational symmetry. ${ }^{5}$ Actually, one would expect that the emission of Cherenkov radiation would be suppressed within gap regions of photonic crystals, ${ }^{6}$ and the emission outside the gaps could be interpreted as originating in the coupling of the electron to allowed propagation modes inside the crystal. In this sense, both EELS and the analysis of the emitted light can provide useful information on the band structure of this type of materials. $^{7}$

The purpose of this work is to establish a connection between EELS and the photonic band structure in photonic crystals. Here, we will focus on the case of 2D crystals and electron trajectories that are parallel to the axis of translational symmetry. Then, the excitation frequency $\omega$ and electron velocity $v$ determine the parallel momentum of the relevant radiation modes, $q=\omega / v$, and the response of the crystal can be actually studied separately for each point of the $(q, \omega)$ plane by tuning appropriately the electron velocity.
It is important to emphasize that $q$ is always larger than the momentum of light in vacuum, $\omega / c$, so that electrons offer the possibility to study regions of the $(q, \omega)$ plane below the light cone (i.e., evanescent waves in vacuum) that cannot be easily accessed by external light.

A 2D array of infinitely long cylindrical holes drilled in a dielectric will be investigated in this work as a characteristic example of photonic crystal. An actual realization of this type of structure is provided by photonic crystal fibers, where cylindrical holes running along optical fibers are made to confine light in the core region for gap frequencies of the crystal. $^{8}$

Photonic bands and EELS for broad electron beams are discussed using plane-wave expansions to solve Maxwell's equations in Sec. II, where numerical calculations of loss spectra and electron angular distributions are offered. However, plane waves are not adequate to describe localized trajectories and fail to converge for focused beams. This problem is solved in Sec. III by using multiple-scattering theory for electrons traveling inside one of the cylinders of the crystal. The results are then compared with finite crystals described within multiple scattering as well.

Gaussian atomic units (i.e., $\hbar=m=e=1$ ) are used throughout the paper unless otherwise specified.

\section{ENERGY LOSS AND ANGULAR DISTRIBUTION IN BROAD ELECTRON BEAMS}

A 2D periodic array of infinitely long parallel cylindrical air cavities drilled in a host medium of dielectric function $\epsilon_{h}$ will be considered in what follows. The electrons will be assumed to move with constant velocity $\mathbf{v}$ parallel to the translational direction of symmetry, taken along the $z$ axis.

When broad beams are employed, electrons can move with all possible impact parameters $\mathbf{b}$ perpendicular to the $z$ axis. A quantum-mechanical treatment of this problem would involve calculating transition probabilities between plane waves representing the fast electrons. However, this has been shown to be fully equivalent to describing the electrons by point charges that follow classical trajectories and then aver- 
aging over $2 \mathrm{D}$ impact parameters $\mathbf{b} .{ }^{9}$ This is the scheme that we will follow here.

A natural representation for this type of beam consists in expanding the electromagnetic field in terms of plane waves. Plane-wave expansions have been intensively used to study photonic band structures. ${ }^{10,11}$ A previous analysis will be extended here to include the external charge of the electron. One can start from Maxwell's equations to show that the magnetic field obeys the equation

$$
\nabla \times\left(\frac{1}{\epsilon} \nabla \times \mathbf{H}\right)-\frac{\omega^{2}}{c^{2}} \mathbf{H}=\frac{4 \pi}{c} \nabla \times \frac{1}{\epsilon} \mathbf{j}
$$

where $\epsilon(\mathbf{r}, \omega)$ is the position-dependent, local dielectric constant, $\mathbf{j}(\mathbf{r}, t)=-\mathbf{v} \delta(\mathbf{R}-\mathbf{b}) \delta(z-v t)$ is the external current of the electron, and $\mathbf{r}=(\mathbf{R}, z)$. In frequency space $\omega$, the current $\mathbf{j}$ can be recast as

$$
\mathbf{j}(\mathbf{r}, \omega)=-\hat{\mathbf{z}} \sum_{\mathbf{G}_{0}} \int_{1 \mathrm{BZ}} \frac{d^{2} \mathbf{Q}}{(2 \pi)^{2}} e^{\mathrm{i}\left(\mathbf{Q}+\mathbf{G}_{0}\right) \cdot(\mathbf{R}-\mathbf{b})} e^{\mathrm{i} q z},
$$

where $\mathbf{G}_{0}$ runs over $2 \mathrm{D}$ reciprocal lattice vectors of the crystal under consideration, $q=\omega / v$ is the momentum of the radiation along the $z$ direction, and the integral is extended over the first Brillouin zone (1BZ). The linearity of Maxwell's equations permits solving each $\mathbf{Q}+\mathbf{G}_{0}$ component separately. Then, invoking Bloch's theorem in virtue of the periodicity of $\epsilon(\mathbf{r}, \omega)$, one can show that the transversal field $\mathbf{H}$ admits the expansion

$$
\mathbf{H}=\sum_{\mathbf{G}} \sum_{\sigma=s, p} h_{\mathbf{G}}^{\sigma} \hat{b}_{\sigma} e^{\mathrm{i}(\mathbf{Q}+\mathbf{G}) \cdot \mathbf{R}} e^{\mathrm{i} q z},
$$

where $\hat{b}_{s}=(\cos \theta \cos \varphi, \cos \theta \sin \varphi,-\sin \theta)$ and $\hat{b}_{p}=(-\sin \varphi$, $\cos \varphi, 0)$ form a set of orthogonal vectors together with $(\mathbf{Q}$ $+\mathbf{G}, q)$, and $(\theta, \varphi)$ are the polar angles of the latter. Inserting this expression into Eq. (1), one obtains ${ }^{10}$

$$
\begin{gathered}
\sum_{\mathbf{G}^{\prime}}\left\{\left[\begin{array}{cc}
\mathrm{N}_{\mathbf{G G}^{\prime}}^{p p} & \mathrm{~N}_{\mathbf{G G}^{\prime}}^{p s} \\
\mathrm{~N}_{\mathbf{G G}^{\prime}}^{s p} & \mathrm{~N}_{\mathbf{G} \mathbf{G}^{\prime}}^{s s}
\end{array}\right]-\frac{\omega^{2}}{c^{2}} \delta_{\mathbf{G}^{-} \mathbf{G}^{\prime}}\right\}\left[\begin{array}{c}
h_{\mathbf{G}^{\prime}}^{p} \\
h_{\mathbf{G}^{\prime}}^{s}
\end{array}\right] \\
=\frac{4 \pi \mathrm{i}}{c} \epsilon_{\mathbf{G}, \mathbf{G}_{0}}^{-1}|(\mathbf{Q}+\mathbf{G}, q)|\left[\begin{array}{c}
-\sin \theta \\
0
\end{array}\right],
\end{gathered}
$$

where the elements of $\mathrm{N}$ are

$$
\begin{gathered}
\mathrm{N}_{\mathbf{G G}^{\prime}}^{p p}=F\left[\cos \theta \cos \theta^{\prime} \cos \left(\varphi-\varphi^{\prime}\right)+\sin \theta \sin \theta^{\prime}\right], \\
\mathbf{N}_{\mathbf{G G}^{\prime}}^{p s}=-F \cos \theta \sin \left(\varphi-\varphi^{\prime}\right), \\
\mathrm{N}_{\mathbf{G G}^{\prime}}^{s p}=F \cos \theta^{\prime} \sin \left(\varphi-\varphi^{\prime}\right), \\
\mathrm{N}_{\mathbf{G} \mathbf{G}^{\prime}}^{s s}=F \cos \left(\varphi-\varphi^{\prime}\right), \\
F=\epsilon_{\mathbf{G}, \mathbf{G}^{\prime}}^{-1}\left|(\mathbf{Q}+\mathbf{G}, q) \|\left(\mathbf{Q}+\mathbf{G}^{\prime}, q\right)\right|
\end{gathered}
$$

$\left(\theta^{\prime}, \varphi^{\prime}\right)$ are the polar angles of $\left(\mathbf{Q}+\mathbf{G}^{\prime}, q\right)$, and $\epsilon_{\mathbf{G}, \mathbf{G}^{\prime}}^{-1}$ is the inverse matrix of the Fourier transform of $\epsilon(\mathbf{r}, \omega)$. In our specific geometry, one has

$$
\epsilon_{\mathbf{G}, \mathbf{G}^{\prime}}=\boldsymbol{\epsilon}_{h} \delta_{\mathbf{G}, \mathbf{G}^{\prime}}+\frac{2 \pi\left(1-\boldsymbol{\epsilon}_{h}\right)}{\left|\mathbf{G}-\mathbf{G}^{\prime}\right|^{2} A} \int_{0}^{\left|\mathbf{G}-\mathbf{G}^{\prime}\right| D / 2} \zeta d \zeta J_{0}(\zeta)
$$

where $A$ is the unit cell area and $D$ is the diameter of the cylindrical holes.

The eigenvalues of $\mathrm{N}$ correspond to the values of $\omega^{2} / c^{2}$ for which light can propagate inside the crystal, that is, the photonic band structure. This is shown in Fig. 1(a) for air holes in $\mathrm{Si}\left(\epsilon_{h}=11.9\right)$ with a diameter to lattice constant ratio $D / a=0.8$. The region actually probed by the electron lies in the $\omega=q v$ plane, which has been represented as well for $v=0.7 c$. These results, as well as the loss probability discussed below for broad beams, have converged using a few hundred plane waves.

The energy loss experienced by the electron can be calculated from the work exerted by the induced electric field acting back on the probe. Integrating this quantity over the electron trajectory $\mathbf{r}_{e}(t)=(\mathbf{b}, v t)$, the energy loss $\Delta E$ can be written $^{12}$

$$
\Delta E=\int d t \mathbf{v} \cdot \mathbf{E}^{\mathrm{ind}}\left[\mathbf{r}_{e}(t), t\right]=L \int_{0}^{\infty} \omega d \omega P(\omega),
$$

where $L$ is the length of the trajectory and

$$
P(\omega)=\frac{1}{\pi \omega L} \int d t \operatorname{Re}\left\{e^{-\mathrm{i} \omega t} \mathbf{v} \cdot \mathbf{E}^{\mathrm{ind}}\left[\mathbf{r}_{e}(t), \omega\right]\right\}
$$

is the electron energy loss probability per unit of path length. Only the $z$ component of the induced field is needed, which can be derived from Ampère's law to yield an expansion similar to Eq. (3) in which the $\mathbf{G}$ component reads

$$
\begin{aligned}
\hat{\mathbf{z}} \cdot \mathbf{E}_{\mathbf{G G}_{0}}= & \frac{-1}{\omega / c}\left[\sum_{\mathbf{G}^{\prime}} \epsilon_{\mathbf{G}, \mathbf{G}^{\prime}}^{-1} h_{\mathbf{G}^{\prime}}^{p}\left|\left(\mathbf{Q}+\mathbf{G}^{\prime}, q\right)\right| \sin \theta^{\prime}\right. \\
& \left.+\frac{4 \pi \mathrm{i}}{c} \epsilon_{\mathbf{G}, \mathbf{G}_{0}}^{-1}\right] .
\end{aligned}
$$

This is actually the total field, since the contribution of the bare electron field to the loss probability cancels out in the formula given below. Notice that only $p$ components of $\mathbf{E}$ contribute to the loss. Now, inserting this result into Eq. (4) and summing over all $\mathbf{Q}$ and $\mathbf{G}_{0}$ components as we did in Eq. (2), the loss probability per unit of path length is found to be

$$
P(\omega)=\frac{-1}{4 \pi^{3} \omega} \sum_{\mathbf{G G}} \int_{1 \mathrm{BZ}} d^{2} \mathbf{Q} \operatorname{Re}\left\{\hat{\mathbf{z}} \cdot \mathbf{E}_{\mathbf{G G}_{0}} e^{\mathrm{i}\left(\mathbf{G}-\mathbf{G}_{0}\right) \cdot \mathbf{b}}\right\} .
$$

Finally, the average over 2D impact parameters $\mathbf{b}$ can be easily performed to yield

$$
\bar{P}(\omega)=\int_{1 \mathrm{BZ}} d^{2} \mathbf{Q} \bar{P}(\mathbf{Q}, \omega),
$$

where 


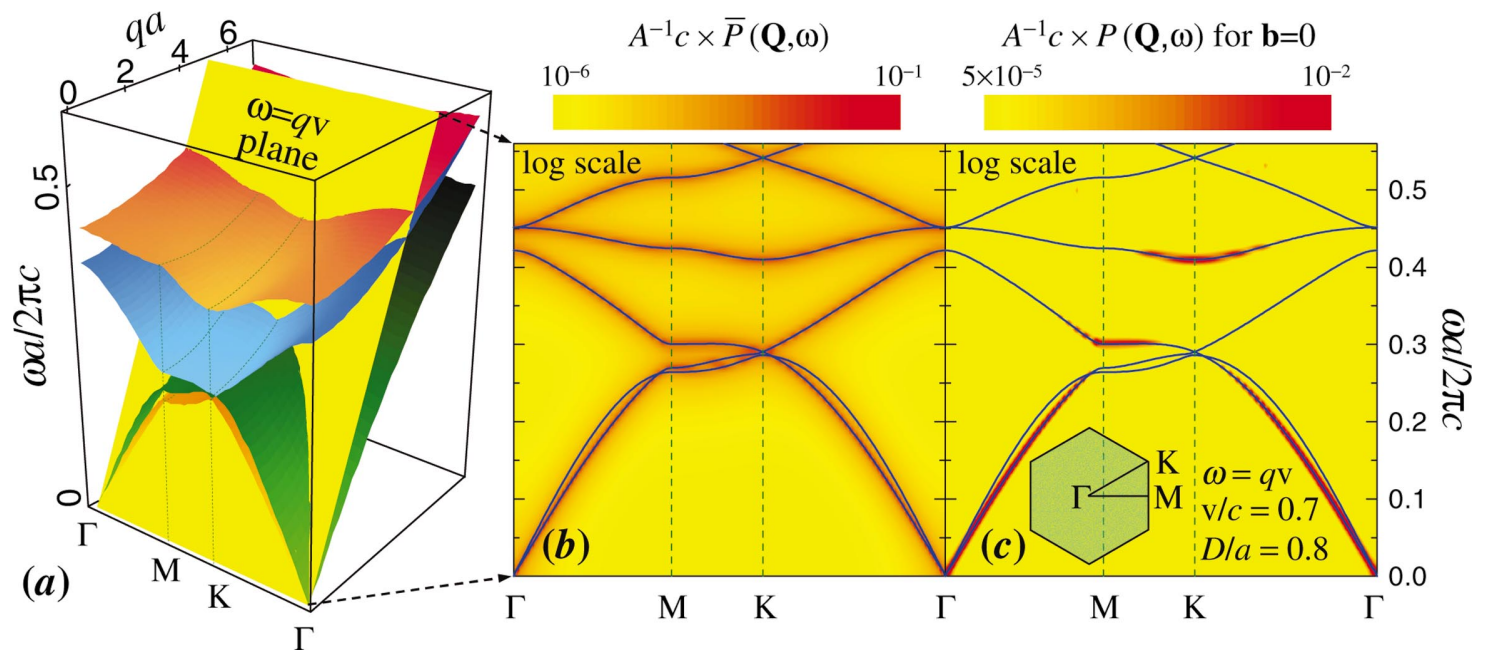

FIG. 1. (Color) (a) Photonic band structure of a triangular 2D crystal made of cylindrical air holes drilled in silicon $\left(\epsilon_{h}=11.9\right)$ for a diameter to lattice constant ratio $D / a=0.8 . q$ is the component of the photon momentum parallel to the cylinders axes. Only the four lowest bands are displayed for the sake of clarity. (b) Intersection of these bands with the $\omega=q v$ plane for $v=0.7 c$ (solid blue curves). The underlying density plot shows the loss probability $\bar{P}(\mathbf{Q}, \omega)$ averaged for all possible electron impact parameters as a function of both photon frequency $\omega$ and momentum transfer perpendicular to the cylinders axes, $\mathbf{Q}$, along the excursion $\Gamma M K \Gamma$. A small imaginary part equal to 0.1 i has been added to $\epsilon_{h}$ in the calculation of $\bar{P}$ in order to improve readability. (c) Similar to (b), but the density plot represents now the loss probability resolved in perpendicular momentum $[P(\mathbf{Q}, \omega)]$ for a focused electron moving along the axis of one of the cylinders. It should be noted that the momentum spread necessary to focus the electron inside one of the holes is larger than the inverse of the diameter, so in realistic experiments one would use broad beams with minimum momentum spread, and the electrons would pass only through the holes giving rise to distributions upon transmission somewhere between (b) and (c).

$$
\bar{P}(\mathbf{Q}, \omega)=\frac{-1}{4 \pi^{3} \omega} \sum_{\mathbf{G}} \operatorname{Re}\left\{\hat{\mathbf{z}} \cdot \mathbf{E}_{\mathbf{G G}}\right\}
$$

is the averaged loss probability resolved in lost energy $\omega$ and perpendicular-momentum transfer $\mathbf{Q}$.
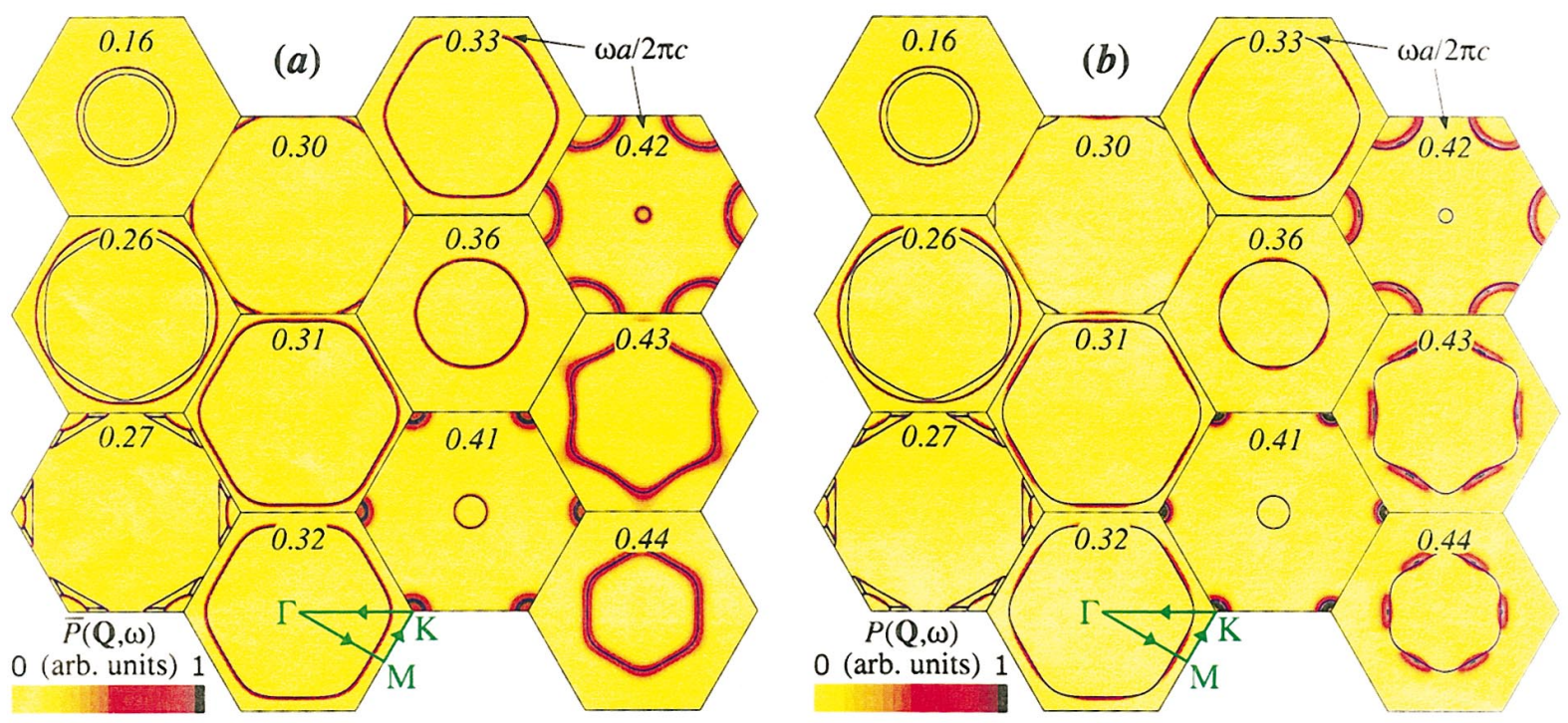

FIG. 2. (Color) (a) Comparison of the photonic band structure (solid blue curves) and the electron energy loss probability averaged over impact parameters, $\bar{P}(\mathbf{Q}, \omega)$, under the same conditions as in Fig. 1(b). Each hexagon represents the first Brillouin zone for a given value of $\omega$, as shown by labels. Notice that the perpendicular-momentum transfer $\mathbf{Q}$ is proportional to the electron deflection angle, and therefore, these plots represent the actual angular distributions of transmitted electrons for specific energy losses in a broad beam configuration. (b) Same as (a), but the density plot represents now the loss probability resolved in perpendicular momentum $[P(\mathbf{Q}, \omega)]$ for a focused electron moving along the axis of one of the cylinders. 


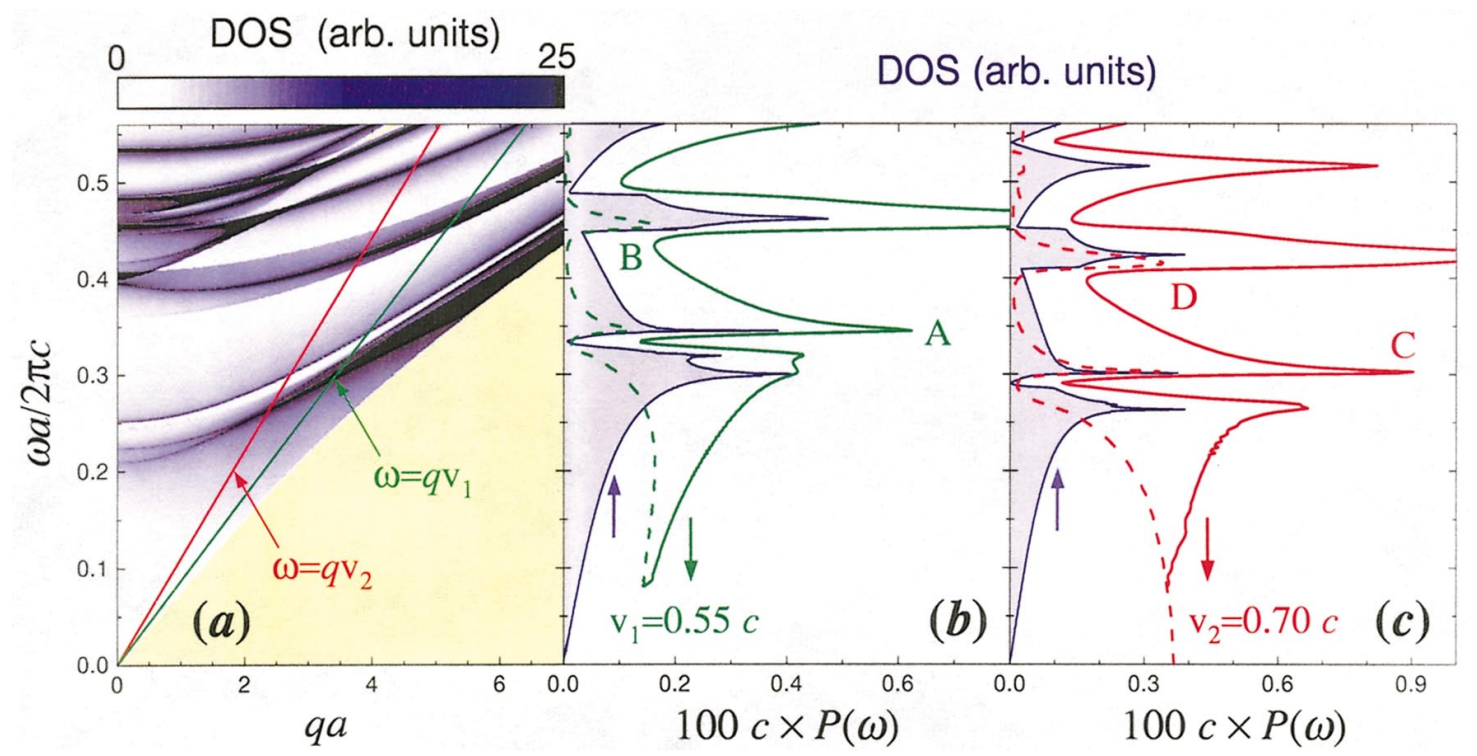

FIG. 3. (a) (Color) Photonic density of states (DOS) of the crystal considered in Fig. 1(a) as a function of photon energy $\omega$ and parallel momentum $q$. The DOS is strictly zero in the lower yellow region. (b) Energy loss probability for a 100 -keV electron $(v=0.55 c)$ moving along the axis of one of the pores in this crystal, as compared to the DOS along the $\omega=q v$ line (shaded area). The dashed curve represents the loss probability $P(\omega)$ for a localized axial trajectory, whereas the solid curve stands for the probability in the case of a broad electron beam, $\bar{P}(\omega)$. A small imaginary part equal to $0.1 \mathrm{i}$ has been added to $\epsilon_{h}$ in order to improve readability. (c) Same as (b) for 200-keV electrons $(v=0.7 c)$.

tonic crystals, this measurable quantity provides the key to directly sampling photonic bands, as shown in Fig. 1(b), where $\bar{P}(\mathbf{Q}, \omega)$ is represented for the crystal of Fig. 1(a) (density plot) and compared with the band structure within the $\omega=q v$ plane. The band structure curves (blue curves) are closely mapped by the regions of high loss probability. It should be noticed, however, that the second lowest band [i.e., the curves in Figs. 1(b) and 1(c) coming from the intersection of the $\omega=q v$ plane with the green surface in Fig. 1(a)] does not contribute to the energy loss.

A different sampling of the band structure is offered in Fig. 2(a), where each hexagon represents the 1BZ for a fixed value of $\omega$. Again, the band structure and regions of high loss probability are in good agreement, except for the second lowest band, which goes largely unnoticed by the electrons. The reason for this behavior can be found in the fact that, first, this band has a pure $s$ character in the $q=0$ limit, where $s$ and $p$ modes do not mix, ${ }^{11}$ and second, only $p$ components contribute to the loss, as pointed out above. ( $p$ and $s$ polarizations for $q=0$ have also been denoted $E$ and $H$, respectively. ${ }^{11}$ )

When the host material is transparent, all energy losses must be converted into radiation, so that $P(\omega)$ can be also interpreted as a light emission probability. This can be connected to recent studies of light emission induced by fast electrons used to characterize collective Mie excitations of nanoparticles. ${ }^{14}$ Therefore, $P(\omega)$ can be obtained not only via EELS but also by measuring photon emission spectra induced by the passage of the electrons.

$\bar{P}(\omega)$ is shown in Fig. 3 for electrons moving at two different velocities in the same crystal as in Fig. 1 [solid curves in Figs. 3(b) and 3(c)]. The photonic density of states
(DOS) is given for comparison in Fig. 3(a), as calculated using the procedure described in Ref. 11. It shows gaps (white regions) and intense maxima (black regions) that are sampled by the electrons along the $\omega=q v$ lines for the two velocities under consideration. The loss spectra are compared with the DOS along these lines [shaded areas in Figs. 3(b) and $3(\mathrm{c})]$. The features of the loss spectra follow quite closely those of the DOS. In particular, this is the case of peaks $A-D$, which can be interpreted as originating in the coupling of the electron to modes that contribute to DOS peaks at frequencies such that some bands cross the $K$ and $M$ points, where they are quite flat, so that the DOS is strongly enhanced.

The lowest band in Fig. 1(a) gives rise to a smooth but relatively high probability in the low- $\omega$ region of Figs. 3(b) and 3(c). Actually, the light cannot resolve the holes in the $\omega \rightarrow 0$ limit, so that these energy losses must be interpreted as originating in the emission of Cherenkov radiation within an effective homogeneous medium. Finally, some bands do not couple to the electron, like the second lowest band (see above), which introduces a peak in the DOS but does not affect $\bar{P}(\omega)$.

\section{ENERGY LOSS IN FOCUSED BEAMS}

The convergence of the above plane-wave formalism is slower when one considers an electron beam that is focused inside one of the cylindrical holes. Then, the interaction of the electron with the photonic crystal becomes small, so that contributions to the loss probability coming from spurious host components are dominant, unless a large number of plane waves is used. Convergence for broad beams was 
achieved with 61 plane waves in the previous section, whereas focused beams take around 151 waves for the examples under consideration; that is, the computation of the involved matrix inversions is 15 times slower.

However, this aloof configuration has practical importance, since electrons will be able to move with minimum loss along large distances inside the selected hole. Actually, an analysis of an experiment that uses this type of geometry has been recently given by the authors with no details of the calculation, ${ }^{15}$ so that we will develop the required theory next, along with some examples.

Multiple scattering will be used to speed the calculation for focused beams. The electric field within each medium $j$ ( $j=1$ for air and $j=h$ for the host material) will be expanded in terms of outgoing and standing cylindrical waves, $\mathbf{E}_{j, q m \sigma}^{H}(\mathbf{r}, \omega)$ and $\mathbf{E}_{j, q m \sigma}^{J}(\mathbf{r}, \omega)$, respectively, where $\sigma(=s$ or $p$ ) is the polarization and $m$ runs over azimuthal quantum numbers. Detailed expressions for this type of waves are given in Appendix A. In practice, convergence is achieved for $m \leqslant 4$, which results in $18 \times 18$ secular matrices, much smaller as compared with the plane-wave expansion formalism. Nevertheless, the latter is still convenient for broad beams, since it permits integrating over impact parameters analytically, as shown in Sec. II.

First of all, we shall consider a single cylindrical cavity inside of which the electron is moving. The external field of the electron can be expressed in terms of $p$-polarized cylindrical waves, as shown in Appendix A. Near the cylinder boundary, the bare electron field is composed of outgoing waves, as described by Eq. (A5). These waves are reflected at the cylinder boundary to produce standing waves inside the cylinder, so that the electric field can be written, in $(q, \omega)$ space, as

$$
\mathbf{E}=\sum_{m}\left[\psi_{m}^{\mathrm{ext}} \mathbf{E}_{1, q m p}^{H}+\sum_{\sigma} \psi_{m \sigma}^{\mathrm{ref}} \mathbf{E}_{1, q m \sigma}^{J}\right]
$$

within that region, whereas the electric field outside the cylinder consists of transmitted outgoing waves,

$$
\mathbf{E}=\sum_{m \sigma} \psi_{m \sigma}^{0} \mathbf{E}_{h, q m \sigma}^{H} .
$$

Here, $\psi_{m}^{\text {ext }}$ are the coefficients of the expansion of Eq. (A5) [see Eq. (A6)], whereas $\psi_{m \sigma}^{0}$ and $\psi_{m \sigma}^{\text {ref }}$ are determined by the boundary conditions at the cylinder wall according to Eq. (B3) of Appendix B.

For crystals we will use a 2D Korringa-Kohn-Rostoker (KKR) description ${ }^{16}$ consisting in expressing the electric field inside the host medium in terms of cylindrical waves centered around the cylinders:

$$
\mathbf{E}(\mathbf{r})=\sum_{\alpha} \sum_{m \sigma} \psi_{\alpha, m \sigma} \mathbf{E}_{h, q m \sigma}^{H}\left(\mathbf{R}-\mathbf{R}_{\alpha}\right),
$$

where $\mathbf{R}_{\alpha}$ runs over 2D lattice sites. Outgoing cylindrical waves $\mathbf{E}_{h, q m \sigma}^{H}$ have been chosen in order to guarantee that the electric field has the correct far-field behavior.

The coefficients $\psi_{\alpha, m \sigma}$ can be determined from the selfconsistent interaction among cylinders. More precisely, the outgoing waves centered around each cylinder $\alpha^{\prime} \neq \alpha$ can be translated to a given cylinder $\alpha$, where they behave like passing standing waves. These waves are in turn scattered by cylinder $\alpha$, giving rise to outgoing waves that add up to the coefficients $\psi_{\alpha, m \sigma}$. Putting this together, in a way similar to standard techniques of multiple-scattering theory, ${ }^{17}$ the following self-consistent relation is obtained:

$$
\psi_{\alpha}=\psi_{\alpha}^{0}+t \sum_{\alpha^{\prime} \neq \alpha} T_{\alpha \alpha^{\prime}} \psi_{\alpha^{\prime}}
$$

where matrix notation has been used such that $\psi_{\alpha}$ represents the vector of components $\psi_{\alpha, m \sigma}$, and matrix-vector products involve a sum over $(m \sigma)$ indices. The first term on the righthand side of this equation stands for the field components in the absence of multiple scattering, $\psi_{\alpha}^{0}$. The remaining sum is the contribution to the field in site $\alpha$ coming from the electric field propagated from every other site $\alpha^{\prime}$ to $\alpha$ by means of the translation matrix $T_{\alpha \alpha^{\prime}, m \sigma, m^{\prime} \sigma^{\prime}}=\delta_{\sigma \sigma^{\prime}} T_{m^{\prime}-m}^{\alpha \alpha^{\prime}}$ [see Eq. (A4) in Appendix A for explicit expressions of $\left.T_{m^{\prime}-m}^{\alpha \alpha^{\prime}}\right]$ and scattered at $\alpha$ via the cylinder scattering matrix $t_{m \sigma, m^{\prime} \sigma^{\prime}}$ $=\delta_{m m^{\prime}} t_{m, \sigma \sigma^{\prime}}$ [a detailed derivation of $t_{m, \sigma \sigma^{\prime}}$ is offered in Appendix B; see Eqs. (B1) and (B2) in particular].

The translational symmetry of the crystal permits writing the electric field components in the 2D perpendicular momentum representation $\mathbf{Q}$ as

$$
\psi_{\alpha}=\frac{A}{(2 \pi)^{2}} \int_{1 \mathrm{BZ}} d^{2} \mathbf{Q} \psi_{\mathbf{Q}} e^{\mathrm{i} \mathbf{Q} \cdot \mathbf{R}_{\alpha}},
$$

which admits the inverse Fourier transformation

$$
\psi_{\mathbf{Q}}=\sum_{\alpha} \psi_{\alpha} e^{-\mathrm{i} \mathbf{Q} \cdot \mathbf{R}_{\alpha}}
$$

Inserting this expressions into Eq. (7), one finds that different Q components are decoupled. More precisely,

$$
\psi_{\mathbf{Q}}=\frac{1}{1-t T_{\mathbf{Q}}} \psi_{\mathbf{Q}}^{0},
$$

where a $\mathbf{Q}$-dependent translation matrix has been defined as

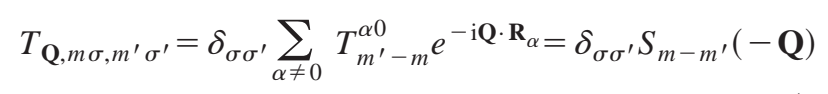

and one has chosen $\mathbf{R}_{0}=0$. Analytical expressions for the lattice sum $S_{m}(\mathbf{Q})$ are given in Appendix C.

The inhomogeneous part of Eq. (7) takes the form $\psi_{\alpha, m \sigma}^{0}=\delta_{\alpha 0} \psi_{m \sigma}^{0}$, where $\alpha=0$ has been chosen as the cylinder inside of which the electron is moving and $\psi_{m \sigma}^{0}$ is given by Eq. (B3) for the electron source under consideration [see Eq. (6) as well]. Then one has, according to Eq. (9), $\psi_{\mathbf{Q}, m \sigma}^{0}$ $=\psi_{m \sigma}^{0}$, which is independent of $\mathbf{Q}$.

The induced electric field near the electron trajectory is made up of the result of internal scattering inside the cylinder, as described by $\psi_{m \sigma}^{\mathrm{ref}}$ in Eqs. (5) and (B3), plus the trans- 
mission into the $\alpha=0$ cylinder of outgoing waves coming from the rest of the cylinders. One finds

$$
\mathbf{E}^{\mathrm{ind}}=\sum_{m \sigma} \mathbf{E}_{1, q m \sigma}^{J}\left\{\psi_{m \sigma}^{\mathrm{ref}}+\sum_{\sigma^{\prime}} A_{m, \sigma \sigma^{\prime}}\left[\sum_{\alpha \neq 0} T_{0 \alpha} \psi_{\alpha}\right]_{m \sigma^{\prime}}\right\},
$$

where $A_{m, \sigma \sigma^{\prime}}$ represents the transmission coefficient of a standing wave into the cylinder inner region, as defined by Eqs. (B1) and (B2), and $\psi_{\alpha}$ is the solution of Eq. (7).

Finally, the energy loss probability per unit of path length is calculated by inserting Eq. (12) into Eq. (4). Here $P(\omega)$ can be decomposed as

$$
P(\omega)=P_{1}(\omega)+P_{c}(\omega),
$$

where

$$
P_{1}(\omega)=\sum_{m} \operatorname{Re}\left\{\frac{\epsilon_{1}}{\left(\pi k_{1}\right)^{2}} \psi_{m}^{\mathrm{ext}} \psi_{m p}^{\mathrm{ref}}\right\}
$$

is the loss probability in the presence of a single cylindrical cavity (see Ref. 18 for more detailed expressions),

$$
P_{c}(\omega)=\sum_{m \sigma^{\prime}} \operatorname{Re}\left\{\frac{\epsilon_{1}}{\left(\pi k_{1}\right)^{2}} \psi_{m}^{\mathrm{ext}} A_{m, p \sigma^{\prime}}\left[\sum_{\alpha \neq 0} T_{0 \alpha} \psi_{\alpha}\right]_{m \sigma^{\prime}}\right\}
$$

is the contribution to the loss probability originating in the rest of the crystal, and $\psi_{m}^{\text {ext }}$ is defined by Eq. (A6).

Using Eq. (8), one can write

$$
P(\omega)=\int_{1 \mathrm{BZ}} d^{2} \mathbf{Q} P(\mathbf{Q}, \omega),
$$

where

$$
\begin{aligned}
P(\mathbf{Q}, \omega)= & \frac{A}{(2 \pi)^{2}} \sum_{m} \operatorname{Re}\left\{\frac{\epsilon_{1}}{\left(\pi k_{1}\right)^{2}} \psi_{m}^{\mathrm{ext}}\right. \\
& \left.\times\left[\psi_{m p}^{\mathrm{ref}}+\sum_{m^{\prime} \sigma^{\prime}} A_{m, p \sigma^{\prime}} S_{m-m^{\prime}}(-\mathbf{Q}) \psi_{\mathbf{Q}, m^{\prime} \sigma^{\prime}}\right]\right\}
\end{aligned}
$$

is the crystal contribution revolved in perpendicular momentum $\mathbf{Q}$ and $\psi_{\mathbf{Q}, m \sigma}$ is the solution of the multiple-scattering equations as given by Eq. (10).

$P(\mathbf{Q}, \omega)$ is represented in Fig. 1(c) (density plot) and compared with the photonic band structure of the crystal under discussion within the $\omega=q v$ plane (blue curves). The data shown in the figure correspond to an axial trajectory $(\mathbf{b}=0)$, but very similar results are obtained for off-center trajectories. A more reduced region of the band structure is contributing to the loss, as compared with the case of a broad beam [Fig. 1(b)]. In particular, regions near the $M$ and $K$ points at around $\omega a / 2 \pi c=0.3$ and 0.4 , respectively, seem to be emphasized. The effect is very apparent in Fig. 2(b), where $\mathbf{Q}$ maps are presented for selected energy losses. This has direct consequences in the loss spectra shown in Figs. 3 (b) and 3(c). For instance, the loss peaks $A-D$ lie on top of maxima in the DOS for broad beams (solid curves), but peaks $B$ and $D$ occur near a sudden rise of the DOS for localized beams (dashed curves).

The position of the loss peaks with respect to the DOS features can be understood by analyzing the two-dimensional Van Hove singularities (VHS's) associated with our DOS [the third dimension is actually fixed by the condition $\omega$ $=q v$, which applies to all curves in Figs. 3(b)-3(c)]. The effect of VHS's on the electronic density of states has been observed via EELS features assigned to electron interband transitions, ${ }^{19}$ but here we are concerned instead with the photonic density of states.

Van Hove singularities are associated with the vanishing of the group velocity for specific $\mathbf{Q}$ points of photonic bands $n$ [i.e., $\nabla_{\mathbf{Q}} \omega_{n}(\mathbf{Q}, q)=0$ ] ${ }^{20-22}$ This is translated into characteristic features in the density of states, $\operatorname{DOS}(\omega)$ $=\Sigma_{n} \int_{1 \mathrm{BZ}} d^{2} \mathbf{Q} \delta\left[\omega-\omega_{n}(\mathbf{Q}, q)\right]$. In our two-dimensional momentum case, there are two different kinds of VHS's: (i) those coming from either minina or maxima of the bands $\omega_{n}(\mathbf{Q}, q)$, where the DOS becomes discontinuous, and (ii) those arising from saddle points, which yield logarithmic divergences in the DOS. This behavior has to emerge in the loss probability, since it involves an integral over $\mathbf{Q}$ similar to the one in the DOS.

One can clearly observe the presence of saddle points in the band structure near the $M$ point at $\omega a / 2 \pi c \approx 0.3$ and 0.45 in Figs. 1(b)-1(c), which should give rise to VHS's of type (ii) in the DOS [Fig. 3(c)]. Also, there is a minimum at the $K$ point near $\omega a / 2 \pi c \approx 0.41$, which corresponds to VHS's of type (i). Now, for broad beams Fig. 1(b) indicates that these regions of the band structure are sampled by the electrons, so that logarithmic divergences show up in the loss probability at those energies [peaks $C$ and $D$ for $200-\mathrm{keV}$ broad beams in Fig. 3(c)]. However, the saddle point near $\omega a / 2 \pi c \approx 0.45$ is not efficiently sampled by the localized central trajectory of Fig. 1(c), so that no peak appears in the loss probability near that frequency [dashed curve in Fig. 3(c)]. However, the localized trajectory exhibits a feature near $\omega a / 2 \pi c \approx 0.41$ that should be attributed to VHS's of type (i) and that is shadowed by the more intense $\omega a / 2 \pi c \approx 0.45$ peak in the case of the broad beam. The origin of peaks $A$ and $B$ in Fig. 1(b) for $100-\mathrm{keV}$ electrons can be understood in the same way as $C$ and $D$, respectively.

Therefore, EELS features in the localized trajectories under consideration occur in coincidence with VHS's of type (i) in peaks near $B$ and $D$ and of type (ii) in peaks $A$ and $C$. For broad beams, all peaks coincide with VHS of type (ii), and the effect of the VHS of type (i) near $B$ and $D$ is shadowed under dominant type-(ii) VHS's.

In the low-energy end, the spectrum for localized beams converges to the result obtained for broad beams, which is an additional manifestation of Cherenkov radiation for an effective medium whose porous structure cannot be resolved by radiation of much larger wavelength.

Finite-size effects are studied in Fig. 4, where we show the loss probability for crystals composed of a finite number of cylinders. The electron is moving in the central cylinder in all cases. The loss probability has been calculated from Eqs. (13) and (14), where $\psi_{\alpha}$ has been obtained by direct inversion of Eq. (7). ${ }^{23}$ The results have also been reproduced by 


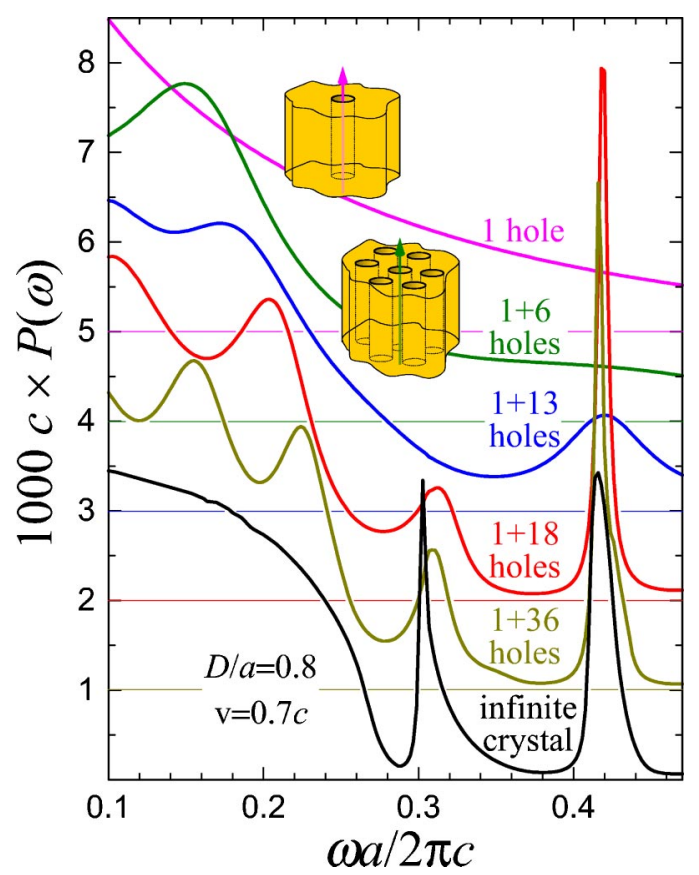

FIG. 4. Energy loss spectra for an electron moving along the axis of a cylindrical hole drilled in silicon $\left(\epsilon_{h}=11.9\right)$ when the hole is isolated (dashed curve) or surrounded by a different number of holes, as indicated by labels. The electron velocity is $v=0.7 c$. The ratio of the cylinder diameter to the lattice constant is $D / a=0.8$. A small imaginary part equal to $0.1 \mathrm{i}$ has been added to $\epsilon_{h}$ and consecutive curves have been shifted upwards by $1000 c P=1$ in order to improve readability.

means of the boundary element method, ${ }^{24}$ in which the electromagnetic field is expressed in terms of discretized boundary charges and currents that are solved self-consistently by imposing the customary boundary conditions. Starting from the single hole, characterized by a smooth Cherenkov radiation spectrum with no salient features, one can observe that a relatively large number of cylinders is necessary to approach the results of the infinite crystal. The peak near $\omega a / 2 \pi c$ $\approx 0.4$ is the first to converge (e.g., for $1+18$ cylinders), and this is consistent with the fact that this is a relatively higherenergy feature where diffusive propagation is achieved for a smaller number of cylinders. The low-energy region of the spectrum takes considerably more cylinders to converge and it exhibits size-dependent oscillations with $\omega$, suggesting that successive reflections at the different layers of cylinders around the central one are taking place.

\section{CONCLUSIONS}

The central message of this work is that fast electrons can be used to probe photonic crystal band structures. This has been shown for electrons moving along the translational direction of symmetry of 2D crystals both for broad electron beams and for localized beams.

Some of the features of the band structure are directly translated into the electron loss spectra. In particular, the loss probability follows to some extent the profile of the photonic density of states in the case of broad beams [see solid curves in Figs. 3(b) and 3(c)].
The energy loss probability has been separated into the contribution of different momentum transfers perpendicular to the trajectories. This quantity can be directly measured from the angular distribution of broad electron beams transmitted through the crystal for selected energy losses, and it provides a direct mapping of the band structure as shown in Figs. 1(b) and 2(a).

The loss probability presents some peaks that can be associated to Van Hove singularities of different types: saddle points, giving rise to logarithmic divergences, and band maxima and minima, which produce smoother features. These types of peaks are sampled differently by localized electrons and broad beams. Measuring the electron deflection angle is not possible for localized beams transmitted trough cylindrical holes in these crystals, since the momentum spread of the incoming beam needed to focus it within the unit cell is of the order of the momentum transfer $(\sim 1 / a)$. However, if the materials involved have real dielectric constants, all losses are converted into emitted light, so that the loss probability can be equally measured from the induced photon emission probability.

Finally, finite crystals have been considered as well. The size of the crystal for which the loss probability approaches the value of the infinite crystal has been shown to depend on the lost energy, and in particular, peaks of higher-energy converge faster as a result of diffusive propagation of the electromagnetic signal far away from the beam.

\section{ACKNOWLEDGMENTS}

The authors gratefully acknowledge help and support from the Basque Departamento de Educación, Universidades e Investigación, the University of the Basque Country UPV/ EHU (Contract No. 00206.215-13639/2001), and the Spanish Ministerio de Ciencia y Tecnología (Contract No. MAT20010946).

\section{APPENDIX A: CYLINDRICAL WAVES}

Analytical expressions. The electric field inside a homogeneous material $j$ free of external charges and currents and characterized by a dielectric function $\epsilon_{j}$ and a magnetic permeability $\mu_{j}$ can be decomposed into cylindrical waves that are labeled by their azimuthal quantum number $m$, the momentum $q$ along a given direction $\hat{\mathbf{z}}$, and the polarization. More explicitly, using cylindrical coordinates $\mathbf{r}=(R, \varphi, z)$,

$$
\mathbf{E}_{j, q m s}^{J}(\mathbf{r})=\left[\frac{\mathrm{i} m}{Q_{j} R} J_{m}\left(Q_{j} R\right) \hat{\mathbf{R}}-J_{m}^{\prime}\left(Q_{j} R\right) \hat{\boldsymbol{\varphi}}\right] e^{\mathrm{i} m \varphi} e^{\mathrm{i} q z}
$$

for $s$ polarization and

$$
\begin{aligned}
\mathbf{E}_{j, q m p}^{J}(\mathbf{r})= & \frac{q}{k_{j}}\left[\mathrm{i} J_{m}^{\prime}\left(Q_{j} R\right) \hat{\mathbf{R}}-\frac{m}{Q_{j} R} J_{m}\left(Q_{j} R\right) \hat{\boldsymbol{\varphi}}\right. \\
& \left.+\frac{Q_{j}}{q} J_{m}\left(Q_{j} R\right) \hat{\mathbf{z}}\right] e^{\mathrm{i} m \varphi} e^{\mathrm{i} q z}
\end{aligned}
$$

for $p$ polarization. Here, $k_{j}=\sqrt{\epsilon_{j} \mu_{j}(\omega / c)^{2}}$, 


$$
Q_{j}=\sqrt{k_{j}^{2}-q^{2}+\mathrm{i} 0^{+}},
$$

the square roots must be chosen to have positive real parts, and the prime denotes differentiation with respect to the argument.

Equations (A1) and (A2) are easily derived from the Hertz vector ${ }^{25} \Pi=\left(0,0, J_{m}\left(Q_{j} R\right)\right) e^{\mathrm{i} m \varphi} e^{\mathrm{i} q z}$ according to $\mathbf{E}_{j, q m s}^{J}=\left(1 / Q_{j}\right) \nabla \times \Pi$ and $\mathbf{E}_{j, q m p}^{J}=\left(1 / k_{j} Q_{j}\right) \nabla \times(\nabla \times \Pi)$. These are transversal waves $\left(\nabla \cdot \mathbf{E}_{j, q m \sigma}^{J}=0\right)$ that satisfy the relation

$$
\mathbf{E}_{j, q m \sigma}^{J}=\frac{1}{k_{j}} \nabla \times \mathbf{E}_{j, q m \sigma^{\prime}}^{J}
$$

for $\sigma \neq \sigma^{\prime}$, which is convenient to obtain the magnetic field from the electric field using Faraday's law, H $=-\left(\mathrm{i} c / \omega \mu_{j}\right) \nabla \times \mathbf{E}$.

Cylindrical waves with real values of $Q=\sqrt{k_{j}^{2}-q^{2}}$ and $Q^{\prime}=\sqrt{k_{j}^{2}-q^{\prime 2}}$ satisfy the following orthogonality relation at $z=0$ :

$$
\begin{gathered}
\int_{0}^{\infty} R d R \int_{0}^{2 \pi} d \varphi \mathbf{E}_{j, q m \sigma}^{J} \cdot\left(\mathbf{E}_{j, q^{\prime} m^{\prime} \sigma^{\prime}}^{J}\right)^{*} \\
=2 \pi \delta_{m m^{\prime}} \delta_{\sigma \sigma^{\prime}} \frac{1}{q} \delta\left(q-q^{\prime}\right) .
\end{gathered}
$$

The above waves are standing in the sense that they do not transport any energy along the radial direction, a property that is reflected in the fact that any combination of these waves results in a Poynting vector whose radial component is zero in a lossless medium. A complete basis of cylindrical waves requires us to define outgoing waves as well, $\mathbf{E}_{j, q m \sigma}^{H}(\mathbf{R})$, which are given by the same expressions as above after substituting the Hankel function $H_{m}^{(1)}$ for the Bessel function $J_{m}$. With this choice, outgoing waves vanish in the far-field limit.

Translation formula. An outgoing wave centered around the position $\mathbf{R}_{\alpha^{\prime}}$ will behave like a combination of standing waves passing by a different position $\mathbf{R}_{\alpha}$. This is actually reflected in the identity

$$
\mathbf{E}_{h, q m^{\prime} \sigma}^{H}\left(\mathbf{R}-\mathbf{R}_{\alpha^{\prime}}\right)=\sum_{m} T_{m^{\prime}-m}^{\alpha \alpha^{\prime}} \mathbf{E}_{h, q m \sigma}^{J}\left(\mathbf{R}-\mathbf{R}_{\alpha}\right)
$$

where

$$
T_{m^{\prime}-m}^{\alpha \alpha^{\prime}}=H_{m^{\prime}-m}^{(1)}\left(Q_{h}\left|\mathbf{R}_{\alpha}-\mathbf{R}_{\alpha^{\prime}}\right|\right) e^{\mathrm{i}\left(m^{\prime}-m\right) \varphi_{\alpha \alpha^{\prime}}},
$$

subject to the condition $\left|\mathbf{R}_{\alpha}-\mathbf{R}_{\alpha^{\prime}}\right|>\left|\mathbf{R}-\mathbf{R}_{\alpha}\right|$. Here, $\varphi_{\alpha \alpha^{\prime}}$ is the azimuthal angle of vector $\mathbf{R}_{\alpha}-\mathbf{R}_{\alpha^{\prime}}$. This can be easily proved from Graf's addition theorem for Bessel functions ${ }^{26}$ and from the detailed form of the cylindrical waves given above. Notice that polarization is conserved during this translation process.

Field of a moving electron. An electron moving inside an infinite, homogeneous medium (say medium 1) along a trajectory parallel to the $z$ axis and described by $R=b, \varphi=0$, and $z=v t$ will set up an electric field that reads, in frequency space $\omega,{ }^{12}$

$$
\begin{aligned}
\mathbf{E}^{\mathrm{ext}}(\mathbf{r}, \omega)= & \int d t e^{\mathrm{i} \omega t} \mathbf{E}^{\mathrm{ext}}(\mathbf{r}, t) \\
= & {\left[\frac{1}{\epsilon_{1}} \nabla-\frac{\mathrm{i} \omega \mu_{1}}{c^{2}} \mathbf{v}\right] \frac{\pi \mathrm{i}}{v} \sum_{m} J_{m}\left(Q_{1} R_{<}\right) } \\
& \times H_{m}^{(1)}\left(Q_{1} R_{>}\right) e^{\mathrm{i} m \varphi} e^{i q z}
\end{aligned}
$$

where $R_{<}=\min \{R, b\}, R_{>}=\max \{R, b\}, Q_{1}$ is the perpendicular momentum given by Eq. (A3), and $q=\omega / v$ is the momentum component of the electron field parallel to the direction of motion. Upon inspection, one finds

$$
\mathbf{E}^{\operatorname{ext}}(\mathbf{r}, \omega)=\sum_{m} \psi_{m}^{\mathrm{ext}} \mathbf{E}_{1, q m p}^{H}(\mathbf{r})
$$

with

$$
\psi_{m}^{\mathrm{ext}}=\frac{\pi Q_{1} k_{1}}{\epsilon_{1} \omega} J_{m}\left(Q_{1} b\right)
$$

for $b \leqslant R$, and a similar expression is obtained for $b>R$. Notice that the field of the electron is entirely composed of $p$ waves.

\section{APPENDIX B: THE SCATTERING MATRIX OF A CYLINDER}

Scattering of external standing waves. The scattering of a standing wave by a cylinder gives rise to scattered outgoing waves. Since outgoing waves diverge at the origin, the field transmitted inside the cylinder has to be a combination of only standing waves. Besides, the azimuthal quantum number $m$ is conserved during scattering due to the symmetry of the cylinder. Thus, the electric field can be written, for fixed values of $\sigma, m, q$, and $\omega$, as

$$
\mathbf{E}=A_{m, s \sigma} \mathbf{E}_{1, q m s}^{J}+A_{m, p \sigma} \mathbf{E}_{1, q m p}^{J}
$$

inside the cylinder, occupied by material 1 , and

$$
\mathbf{E}=\mathbf{E}_{h, q m \sigma}^{J}+t_{m, s \sigma} \mathbf{E}_{h, q m s}^{H}+t_{m, p \sigma} \mathbf{E}_{h, q m p}^{H}
$$

in the surrounding host material $h$, where the first term in the last expression represents the external standing wave of polarization $\sigma$.

Using the expressions (A1) and (A2) of Appendix A for the cylindrical waves and imposing the continuity of the components of the electric field and magnetic field parallel to the cylinder surface (i.e., $\varphi$ and $z$ components; the continuity of the perpendicular $R$ component of both the magnetic induction and the electric displacement is automatically guaranteed by these conditions), one finds 


$$
\left[\begin{array}{c}
A_{m, s s} \\
t_{m, s s} \\
A_{m, p s} \\
t_{m, p s}
\end{array}\right]=M^{-1}\left[\begin{array}{c}
\frac{Q_{h}}{k_{h}} J_{m}\left(a_{h}\right) \\
J_{m}^{\prime}\left(a_{h}\right) \\
0 \\
\frac{m q}{k_{h} a_{h}} J_{m}\left(a_{h}\right)
\end{array}\right]
$$

and

$$
\left[\begin{array}{c}
A_{m, s p} \\
t_{m, s p} \\
A_{m, p p} \\
t_{m, p p}
\end{array}\right]=M^{-1}\left[\begin{array}{c}
0 \\
\frac{m q}{k_{h} a_{h}} J_{m}\left(a_{h}\right) \\
\frac{Q_{h}}{k_{h}} J_{m}\left(a_{h}\right) \\
J_{m}^{\prime}\left(a_{h}\right)
\end{array}\right],
$$

where

$$
M=\left[\begin{array}{llll}
\frac{\zeta Q_{1}}{k_{1}} J_{m}\left(a_{1}\right) & \frac{-Q_{h}}{k_{h}} H_{m}^{(1)}\left(a_{h}\right) & 0 & 0 \\
J_{m}^{\prime}\left(a_{1}\right) & -H_{m}^{(1)^{\prime}}\left(a_{h}\right) & \frac{m q}{k_{1} a_{1}} J_{m}\left(a_{1}\right) & \frac{-m q}{k_{h} a_{h}} H_{m}^{(1)}\left(a_{h}\right) \\
0 & \frac{Q_{1}}{k_{1}} J_{m}\left(a_{1}\right) & \frac{-Q_{h}}{k_{h}} H_{m}^{(1)}\left(a_{h}\right) \\
\frac{\zeta m q}{k_{1} a_{1}} J_{m}\left(a_{1}\right) & \frac{-m q}{k_{h} a_{h}} H_{m}^{(1)}\left(a_{h}\right) & \zeta J_{m}^{\prime}\left(a_{1}\right) & -H_{m}^{(1)^{\prime}}\left(a_{h}\right)
\end{array}\right],
$$

$\zeta=\sqrt{\epsilon_{1} \mu_{h} / \epsilon_{h} \mu_{1}}, a_{j}=Q_{j} a$, and $a$ is the radius of the cylinder. It is important to stress that the scattering by the cylinder mixes $s$ and $p$ components in general, except for $q=0$ or $m=0$.

Scattering of internal outgoing waves. An electron moving inside a cylinder produces outgoing waves according to Eq. (A5), which are scattered at the cylinder boundary to generate an electric field as the one described by Eqs. (5) and (6). Applying the customary boundary conditions for the electromagnetic field (see above), the coefficients of these equations can be expressed in terms of matrix $M$ as

$$
\left[\begin{array}{c}
\psi_{m s}^{\mathrm{ref}} \\
\psi_{m s}^{0} \\
\psi_{m p}^{\mathrm{ref}} \\
\psi_{m p}^{0}
\end{array}\right]=-\psi_{m}^{\mathrm{ext}} M^{-1}\left[\begin{array}{c}
0 \\
\frac{m q}{k_{1} a_{1}} H_{m}^{(1)}\left(a_{1}\right) \\
\frac{Q_{1}}{k_{1}} H_{m}^{(1)}\left(a_{1}\right) \\
\zeta H_{m}^{(1)^{\prime}}\left(a_{1}\right)
\end{array}\right],
$$

where $\psi_{m}^{\text {ext }}$ has been defined in Eq. (A6).

Analytical but quite involved results can be obtained by inversion of the $4 \times 4$ matrix $M$, and in particular, the oscillation modes of the isolated cylinder are derived from the zeros of $\operatorname{det}\{M\} .{ }^{27}$ Instead, we have chosen to carry on this matrix inversion numerically, since the bulk of the computational demand comes from the calculation of the lattice sums discussed next.

\section{APPENDIX C: LATTICE SUMS}

The lattice sum in Eq. (11) can be converted into rapidly converging sums using Ewald's method. For completeness, we quote the results of Leung and Qiu: ${ }^{16}$

$$
\begin{aligned}
S_{m}(\mathbf{Q}) & =\sum_{\mathbf{R}_{\alpha} \neq 0} H_{-m}^{(1)}\left(Q_{h} R_{\alpha}\right) e^{-\mathrm{i} m \varphi_{\alpha}} e^{\mathrm{i} \mathbf{Q} \cdot \mathbf{R}_{\alpha}} \\
& =\frac{2}{\mathrm{i}^{1+m} \pi} D_{m}-\delta_{m 0},
\end{aligned}
$$

where

$$
\begin{gathered}
D_{m}=D_{m}^{(1)}+D_{m}^{(2)}+D_{m}^{(3)}, \\
D_{m}^{(1)}=\frac{2 \pi}{A Q_{h}^{|m|}} \sum_{\mathbf{G}} \frac{|\mathbf{G}+\mathbf{Q}|^{|m|}}{|\mathbf{G}+\mathbf{Q}|^{2}-Q_{h}^{2}} e^{\left(Q_{h}^{2}-|\mathbf{G}+\mathbf{Q}|^{2}\right) / \eta} e^{-\mathrm{i} m \varphi_{\mathbf{G}}}, \\
D_{m}^{(2)}=\left(\frac{-2 \mathbf{i}}{Q_{h}}\right)^{|m|} \sum_{\mathbf{R}_{\alpha} \neq 0} R_{\alpha}^{|m|} e^{\mathrm{i} \mathbf{Q} \cdot \mathbf{R}_{\alpha}} e^{-\mathrm{i} m \varphi_{\alpha}} \\
\times \int_{(1 / 2) \sqrt{\eta}}^{\infty} \frac{d \xi}{\xi} \xi^{2|m|} e^{-R_{\alpha}^{2} \xi^{2}} e^{Q_{h}^{2} / 4 \xi^{2}},
\end{gathered}
$$




$$
D_{m}^{(3)}=\frac{\delta_{m 0}}{2}\left[\gamma+\ln \left(\frac{Q_{h}^{2}}{\eta}\right)+\sum_{n=1}^{\infty} \frac{1}{n n !}\left(\frac{Q_{h}^{2}}{\eta}\right)^{n}\right] \text {, }
$$

$\varphi_{\alpha}$ and $\varphi_{\mathbf{G}}$ are the azimuthal angles of vectors $\mathbf{R}_{\alpha}$ and $\mathbf{G}$, respectively, $\mathbf{R}_{\alpha}$ runs over the sites of the $2 \mathrm{D}$ real-space lattice and $\mathbf{G}$ over the reciprocal lattice, $A$ is the unit cell area, $\gamma=0.5772 \ldots$ is the Euler constant, and the result is independent of $\eta$, which is adjusted in order to speed up the calculation.
*Electronic address: jga@sw.ehu.es

${ }^{1}$ E.D. Palik, Handbook of Optical Constants of Solids (Academic Press, New York, 1985).

${ }^{2}$ D. Ugarte, C. Colliex, and P. Trebbia, Phys. Rev. B 45, 4332 (1992).

${ }^{3}$ J.M. Cowley and J. Winterton, Phys. Rev. Lett. 87, 016101 (2001).

${ }^{4}$ P.A. Cherenkov, Dokl. Akad. Nauk SSSR 2, 451 (1934); I.M. Frank and I. Tamm, ibid. 14, 109 (1937).

${ }^{5}$ C. Luo, M. Ibanescu, S.G. Johnson, and J.D. Joannopoulos, Science 299, 368 (2003).

${ }^{6}$ J. D. Joannopoulos, R. D. Meade, and J. N. Winn, Photonic Crystals: Molding the Flow of Light (Princeton University Press, Singapore, 1995).

${ }^{7}$ F.J. García de Abajo and L.A. Blanco, Phys. Rev. B 67, 125108 (2003); L. A. Blanco and F. J. García de Abajo, Surf. Sci. 532535, 611 (2003).

${ }^{8}$ J.C. Knight and P.St.J. Russell, Science 296, 276 (2002).

${ }^{9}$ R.H. Ritchie and A. Howie, Philos. Mag. A 58, 753 (1988).

${ }^{10}$ K.M. Ho, C.T. Chan, and C.M. Soukoulis, Phys. Rev. Lett. 65, 3152 (1990).

${ }^{11}$ K. Busch and S. John, Phys. Rev. E 58, 3896 (1998).

${ }^{12}$ F.J. García de Abajo, Phys. Rev. B 59, 3095 (1999).

${ }^{13}$ C.H. Chen and J. Silcox, Phys. Rev. Lett. 35, 390 (1975).
${ }^{14}$ N. Yamamoto, K. Araya, and F.J. García de Abajo, Phys. Rev. B 64, 205419 (2001).

${ }^{15}$ F. J. García de Abajo, A. G. Pattantyus-Abraham, N. Zabala, A. Rivacoba, M. O. Wolf, and P. M. Echenique, Phys. Rev. Lett. 91, 143902 (2003)

${ }^{16}$ K.M. Leung and Y. Qiu, Phys. Rev. B 48, 7767 (1993).

${ }^{17}$ F.J. García de Abajo, Phys. Rev. Lett. 82, 2776 (1999).

${ }^{18}$ N. Zabala, A. Rivacoba, and P. M. Echenique, Surf. Sci. 209, 465 (1989).

${ }^{19}$ A.D. Dorneich, R.H. French, H. Müllejans, S. Loughin, and M. Rühle, J. Microsc. 191, 286 (1998).

${ }^{20}$ L. Van Hove, Phys. Rev. 89, 1189 (1953).

${ }^{21}$ N. W. Ashcroft and N. D. Mermin, Solid State Physics (Saunders College Publishing, New York, 1976).

${ }^{22}$ K. Butsch, M. Frank, A. García Martín, D. Hermann, S.F. Mingaleev, M. Schillinger, and L. Tkeshelashvili, Phys. Status Solidi A 197, 637 (2003).

${ }^{23}$ L.M. Li and Z.Q. Zhang, Phys. Rev. B 58, 9587 (1998).

${ }^{24}$ F.J. García de Abajo and A. Howie, Phys. Rev. Lett. 80, 5180 (1998); Phys. Rev. B 65, 115418 (2002).

${ }^{25}$ J. A. Stratton, Electromagnetic Theory (McGraw-Hill, New York, 1941).

${ }^{26}$ Handbook of Mathematical Functions, edited by M. Abramowitz and I. A. Stegun (Dover, New York, 1972).

${ }^{27}$ J.C. Ashley and L.C. Emerson, Surf. Sci. 41, 615 (1974). 